\title{
Effects of Mitochondrial-Targeted Antioxidants on Real-Time Blood Nitric Oxide and Hydrogen Peroxide Release in Acute Hyperglycemic Rats
}

\author{
Matthew L. Bertolet, Michael Minni, Tyler Galbreath, Robert Barsotti, \\ Lindon H. Young, and Qian Chen
}

Division of Research, Department of Bio-Medical Sciences, Philadelphia College of Osteopathic Medicine (PCOM), Philadelphia, PA, 19131, USA

\section{Introduction}

Diabetes and prediabetes are major public health concerns worldwide due to the high risk of developing micro- and macro-vascular complications. Hyperglycemia, the major criteria for diabetes diagnosis, is causally related to the pathogenesis of vascular complications in diabetic patients. An early event during hyperglycemia is vascular endothelial dysfunction. Normally, the vascular endothelium facilitates blood flow principally by releasing endothelial-derived nitric oxide (NO) via vascular endothelial NO synthase (eNOS) in the presence of an essential co-factor, tetrahydrobiopterin $\left(\mathrm{BH}_{4}\right)$. By contrast, acute and chronic hyperglycemia increase oxidative stress and reduce NO bioavailability $[1,2]$. The reduced endothelial-derived NO bioavailability promotes vasoconstrictive, pro-inflammatory, and pro-thrombotic events, initiating inflammation, thereby recruiting leukocytes, resulting in tissue/organ damage (Figure 1). Therefore, reduction of oxidative stress during hyperglycemia will mitigate vascular endothelial dysfunction and organ damage. Crabtree, et al. [1] found that mitochondria-derived superoxide (SO) contributes to hyperglycemia-induced oxidative stress in cultured vascular endothelial cells. Subsequently, the overproduction of SO promotes the oxidation of $\mathrm{BH}_{4}$ to dihydrobiopterin $\left(\mathrm{BH}_{2}\right)$. The reduced $\mathrm{BH}_{4} / \mathrm{BH}_{2}$ ratio leads to $\mathrm{BH}_{2}$, not $\mathrm{BH}_{4}$, binding to oxygenase domain of eNOS, which causes eNOS to shift its product profile from NO to SO [1] (Figure 1). However, the role of mitochondria in acute hyperglycemia-induced oxidative stress and blood NO reduction has not been evaluated in vivo. Recently, our lab showed that mitoquinone (MitoQ) and SS31 (Szeto-Schiller, D-Arg-Dmt-Lys-Phe-Amide) peptide (Figure 2), mitochondriatargeted antioxidants, significantly reduced blood $\mathrm{H}_{2} \mathrm{O}_{2}$ (an index of oxidative stress) and increased blood NO levels in a hind limb ischemia/reperfusion (I/R) animal model [3]. Oxidative stress is also an important cause of reperfusion injury during I/R. Thus, we hypothesize that MitoQ and SS-31 will reduce blood oxidative stress and increase blood NO levels under acute hyperglycemic conditions.

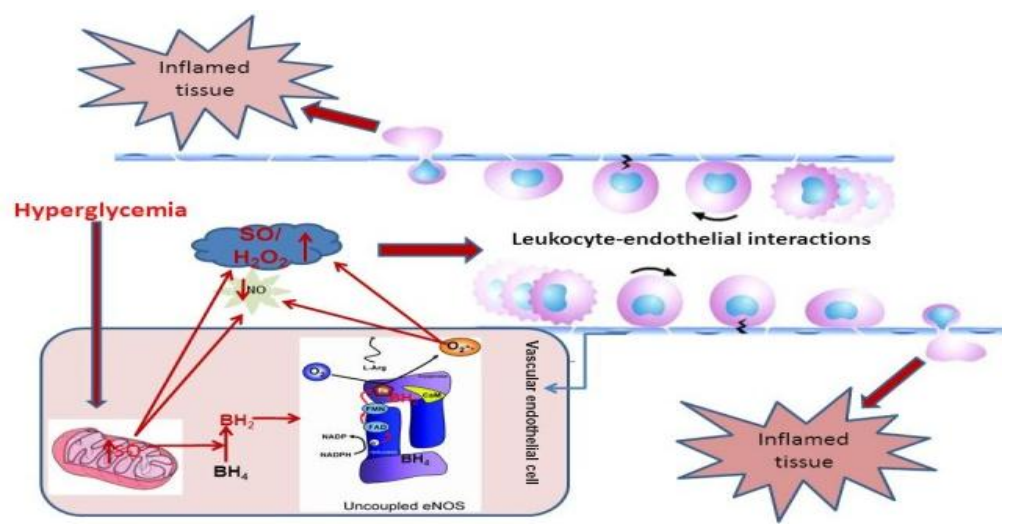

Fig. 1. The putative role of mitochondrial-derived SO in hyperglycemia-induced oxidative stress, vascular endothelial dysfunction and tissue inflammation. 


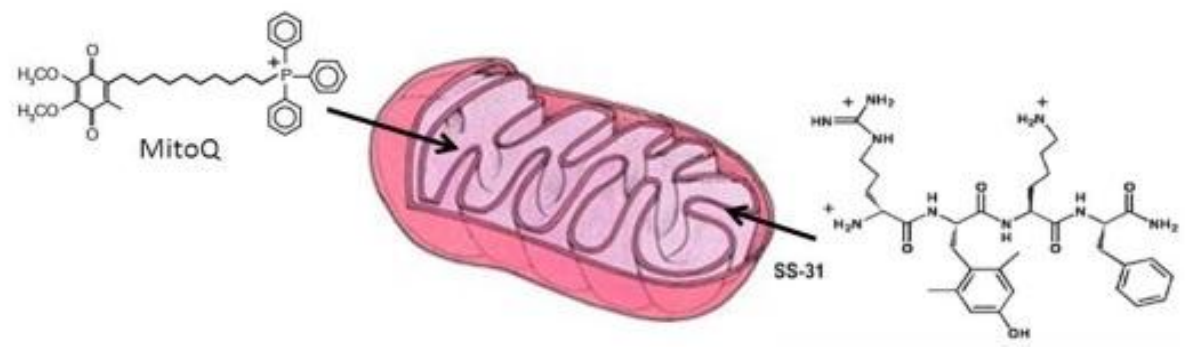

Fig. 2. Chemical structure of Mito $Q$ and SS-31 [4,5].

\section{Results and Discussion}

Male Sprague-Dawley rats (275 to $325 \mathrm{~g}$, Charles River, Springfield, MA) were anesthetized with $60 \mathrm{mg} / \mathrm{kg}$ of pentobarbital sodium with 1000 units of heparin via intraperitoneal (i.p.) injections. The jugular vein was catheterized to allow for the infusion of saline, $20 \% \mathrm{D}$-glucose, or $20 \% \mathrm{D}$-glucose with $1.86 \mathrm{mg} / \mathrm{kg}$ MitoQ (MW=600 g/mol; complexed with cyclodextrin to improve water solubility, total $\mathrm{MW}=1714 \mathrm{~g} / \mathrm{mol}$ ) or with $2.7 \mathrm{mg} / \mathrm{kg}$ SS-31 (MW=640 g/mol, Genemed Synthesis, Inc., San Antonio, TX). The continuous infusion of $20 \% \mathrm{D}$-glucose solution was used to maintain a hyperglycemic state around $200 \mathrm{mg} / \mathrm{dL}$ for about $180 \mathrm{~min}$. MitoQ or SS-31 was added to the $20 \%$ D-glucose infusate to achieve a blood concentration of approximately $13 \mu \mathrm{M}$ and $50 \mu \mathrm{M}$, respectively. Both femoral veins were exposed and catheterized in order to randomly place calibrated $\mathrm{NO}$ or $\mathrm{H}_{2} \mathrm{O}_{2}$ microsensors (100 $\mu \mathrm{m}$ diameter, WPI Inc., Sarasota, FL) into each femoral vein. These microsensors were connected to an Apollo 4000 free radical analyzer (WPI Inc., Sarasota, FL) to monitor blood NO and $\mathrm{H}_{2} \mathrm{O}_{2}$ levels in real-time. $\mathrm{NO}, \mathrm{H}_{2} \mathrm{O}_{2}$ were monitored continuously while glucose levels were recorded at baseline and at 20 minute intervals throughout the 180 minute infusion period [2].

The changes of blood $\mathrm{NO}(\mathrm{nM})$ and $\mathrm{H}_{2} \mathrm{O}_{2}(\mu \mathrm{M})$ levels were expressed as the relative change to the saline group. All the data were represented as a mean \pm SEM, and then analyzed by ANOVA using post hoc analysis with the Student Newman Keuls test. $p<0.05$ was considered significant.

The time course change in blood glucose in different experimental groups is illustrated in Figure 3. The blood glucose level in the saline group remained at baseline values of $80-100 \mathrm{mg} / \mathrm{dl}$ throughout the experiment. In contrast, after $20 \mathrm{~min}$ infusion of $20 \% \mathrm{D}$-glucose with or without the drug, the blood glucose levels rose to hyperglycemic levels at $\sim 200 \mathrm{mg} / \mathrm{dl}$. All hyperglycemic rats urinated between 20-40 min after glucose infusion.

The time course change in blood NO levels relative to the saline infusion among the different experimental groups is shown in Figure 4. There was a significant decrease in blood NO levels in the

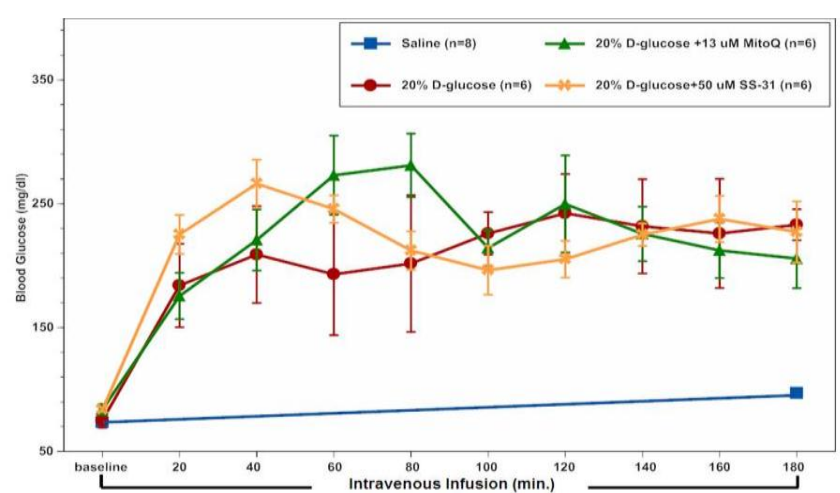

Fig. 3. Blood glucose level among saline and $20 \%$ glucose infusion groups.
$20 \%$ glucose group $(\mathrm{n}=9)$ compared to the saline group $(n=7)$ at $120 \mathrm{~min}$ $(43.7 \pm 11.3 \mathrm{nM}$ lower), followed by a continued decrease throughout the infusion (all $p<0.05$ ). At 180 min, blood NO level was $68 \pm 13.5$ $\mathrm{nM}$ lower relative to that in saline group $(p<0.01)$. However, the coinfusion of MitoQ $(13 \mu \mathrm{M}, \mathrm{n}=5)$ or SS-31 (50 $\mu \mathrm{M} \quad \mathrm{n}=6)$ with $20 \%$ glucose significantly reduced the fall in blood NO levels from 120 min or 140 min, respectively, which was sustained during the rest of the infusion time compared to those in the $20 \%$ glucose group. The NO levels in both MitoQ and SS-31 


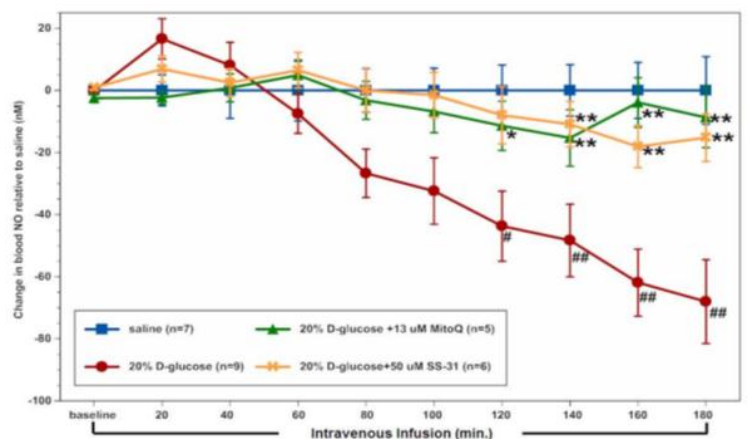

Fig. 4. The comparison of change in blood NO levels relative to saline group among $20 \%$ D-glucose, $20 \%$ D-glucose with Mito Q(13 $\mu M)$, and $20 \%$ D-glucose with SS-31 (50 $\mu M)(\# p<0,05$, \#\#<0.01 vs saline; *p<0,01 vs D-glucose. treatment groups were similar to the NO levels in the saline group throughout the experimental time course.

The time course change in blood $\mathrm{H}_{2} \mathrm{O}_{2}$ levels relative to the saline infusion among the different experimental groups is shown in Figure 5. There was a significant increase in blood $\mathrm{H}_{2} \mathrm{O}_{2}$ levels in the $20 \%$ glucose group $(n=7)$ compared to the saline group $(\mathrm{n}=8)$ at $60 \mathrm{~min}(1.2 \pm 0.3 \mu \mathrm{M}$ higher), followed by a continued increase throughout the infusion. At $180 \mathrm{~min}$, blood $\mathrm{H}_{2} \mathrm{O}_{2}$ level was $3.0 \pm 0.5 \mu \mathrm{M}$ higher relative to that in saline group. However, the addition of SS-31 $(50 \mu \mathrm{M}, \mathrm{n}=5)$ significantly reduced the increase in blood $\mathrm{H}_{2} \mathrm{O}_{2}$ levels from $60 \mathrm{~min}$ to $180 \mathrm{~min}$ compared to those in $20 \%$ glucose group (all $p<0.05$ ). Furthermore, the addition of MitoQ $(13 \mu \mathrm{M}, \mathrm{n}=6)$ significantly reduced

blood $\mathrm{H}_{2} \mathrm{O}_{2}$ levels only at $160 \mathrm{~min}$ and $180 \mathrm{~min}$ by comparing to those in the $20 \%$ glucose group (both $p<0.05)$. SS-31 treatment did show a trend of smaller increases in blood $\mathrm{H}_{2} \mathrm{O}_{2}$ levels compared to MitoQ treatment, but was not significant. Moreover, the $\mathrm{H}_{2} \mathrm{O}_{2}$ levels in both MitoQ and SS-31 treatment groups were not significantly different from $\mathrm{H}_{2} \mathrm{O}_{2}$ levels in the saline group throughout the experimental time course.

Researchers have shown that acute hyperglycemia can cause systemic oxidative stress and vascular dilatory dysfunction in non-diabetic human subjects [6,7]. By placing $\mathrm{NO} / \mathrm{H}_{2} \mathrm{O}_{2}$ sensors in rat femoral veins, we established the time course of blood $\mathrm{NO}$ and $\mathrm{H}_{2} \mathrm{O}_{2}$ levels during acute hyperglycemia. Blood $\mathrm{H}_{2} \mathrm{O}_{2}$ levels serve as an index of oxidative stress because $\mathrm{H}_{2} \mathrm{O}_{2}$ is principally derived from SO by SO dismutase. We found that acute hyperglycemia significantly increased blood $\mathrm{H}_{2} \mathrm{O}_{2}$ and reduced blood NO levels compared to the saline group. Moreover, increased blood $\mathrm{H}_{2} \mathrm{O}_{2}$ levels occurred earlier than reduction of blood NO levels during hyperglycemia. This result suggests that reduced blood NO levels may be due to direct quenching effect of free radicals on blood NO levels and/or eNOS product profile changing from NO to $\mathrm{SO}$ when $\mathrm{BH}_{4}$ is oxidized to $\mathrm{BH}_{2}$ (see Figure 1). Mitochondria may be a site of free radicals overproduction during hyperglycemia. Both MitoQ and

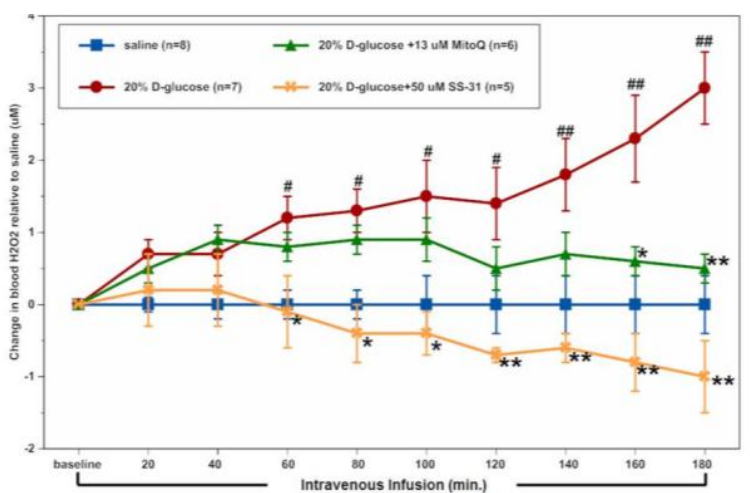

Fig. 5. The comparison of change in blood $\mathrm{H}_{2} \mathrm{O}_{2}$ levels relative to saline group among $20 \% \mathrm{D}$-glucose, $20 \% \mathrm{D}$ glucose with Mito Q (13 um), and 20\% D-glucose with SS-31 (50 uM) (\#p<0.05, \#\#p<0.01 vs saline; *p<0.01 vs D-glucose).
SS-31 have been indicated as mitochondrial-targeted antioxidants by selectively diffusing into the mitochondria after administration [6,7]. We found that administration of MitoQ or SS-31 during hyperglycemia significantly reduced blood $\mathrm{H}_{2} \mathrm{O}_{2}$ levels and improved blood $\mathrm{NO}$ levels, similar to the saline group. Previous research has indicated that MitoQ possesses positive charges and lipophilic properties, and both facilitate its diffusion into the mitochondria. Meanwhile, MitoQ may collapse mitochondrial membrane potential at higher doses which slows its further concentration in the mitochondria [6]. By contrast, SS-31 has an alternating cationic-aromatic amino acid sequence which allows it to concentrate into the mitochondria without relying on mitochondrial membrane potential $[4,5]$. The different mechanism of action 
between MitoQ and SS-31 may explain the observation in this study that SS-31 exerted earlier and better reduction in blood $\mathrm{H}_{2} \mathrm{O}_{2}$ levels compared to MitoQ. In summary, the data suggest that mitochondrial derived SO is a significant source of oxidative stress and vascular endothelial dysfunction under acute hyperglycemic conditions. Moreover, treatment with mitochondrial-targeted antioxidants, MitoQ or SS-31, may be beneficial to attenuate hyperglycemia-induced oxidative stress and vascular endothelial dysfunction.

\section{Acknowledgments}

This study was supported by Division of Research, Department of Bio-Medical Sciences, and Center for Chronic Disorders of Aging at Philadelphia College of Osteopathic Medicine.

\section{References}

1. Crabtree, M.J., et al. Am. J. Physiol. Heart Circ. Physiol. 294, H1530-1540 (2008), http://dx.doi.org/10.1152/ajpheart.00823.2007

2. Minni, M., et al., in Lebl, M. (Ed.) Proceedings of the 22nd American Peptide Symposium, American Peptide Society, San Diego, 2011; 280-281.

3. Galbreath, T., et al., in Lebl, M. (Ed.) Proceedings of the 23rd American Peptide Symposium American Peptide Society, San Diego, 2013, p. 46-47, http://dx.doi.org/10.17952/23APS.2013/046

4. Szeto, H.H. Antioxidants and Redox Signaling 10, 601-619 (2008), http://dx.doi.org/10.1089/ars.2007.1892

5. Adlam, V.J., et al. FASEB J. 19, 1088-1095 (2005), http://www.fasebj.org/content/19/9/1088.full.pdf

6. Title, L.M., et al. J. Am. Coll. Cardiol. 36, 2185-2191 (2000), http://dx.doi.org/10.1016/S07351097(00)00980-3

7. Marfella, R., et al. J. Clin. Invest. 108, 635-636 (2001), http://dx.doi.org/10.1172/JCI13727 